\title{
EDUCACIÓN SANITARIA A LOS ESTUDIANTES DE GRADO EN EDUCACIÓN INFANTIL Y GRADO EN EDUCACIÓN PRIMARIA EN EL MANEJO DEL NIÑO ALÉRGICO EN LA ESCUELA.
}

\author{
SANITARY EDUCATION FOR STUDENTS OF CHILDREN TEACHERS DIRECTED TO DEALING WITH THE \\ ALLERGIC CHILDREN AT THE SCHOOL.
}

$\mathrm{M}^{\mathrm{a}}$ José Barasona Villarejo*, $\mathrm{M}^{\mathrm{a}}$ Luisa Barasona Villarejo, Inmaculada Barranco Cabezudo, Manuel Vaquero Alvarez, Carmen Moreno Aguilar, Eduardo Collantes Estevez.

*mjbarasona@uco.es

Received: 29/06/2018 Accepted: 30/10/2018

\section{Resumen}

La alergia en edad pediátrica es un problema de salud pública emergente y a su vez complejo de abordar. A pesar de esto, apenas hay protocolos de identificación de alumnos alérgicos y tampoco se incluyen en los planes docentes de los futuros profesores aspectos relacionados con la alergia infantil y del adolescente. Dado que los niños pasan una importante parte de su vida en la escuela, con éste proyecto se pretende formar a estudiantes de Grado en Educación Infantil y Grado en Educación Primaria en nociones básicas sobre qué es y qué supone una alergia alimentaria y/ó un asma, en el reconocimiento de los primeros síntomas de una reacción alérgica y en cómo actuar cuando ésta se ha producido.

Se propuso a los participantes (todos alumnos de Magisterio) que rellenaran de forma anónima un cuestionario donde se preguntaba acerca del manejo del niño alérgico en la escuela, mediante 44 preguntas. A continuación se llevarían a cabo varias sesiones formativas y un mes despúes de la formación se volvería a pasar el mismo cuestionario, para valorar la evolución del conocimiento de los alumnos en el tema alergológico.

Con el presente trabajo se muestra que los futuros maestros presentan unos conocimientos escasos acerca de la Alergología. Sería conveniente plantear programas de educación sanitaria de patologías cada vez más prevalentes en la población general y especialmente de las que se manifiestan en niños, ya que como se demuestra en este trabajo, la realización de seminarios informativos mejora de forma significativa el conocimiento sobre dicho tema de los futuros docentes en la escuela.

Palabras clave: Niño, alergia, alimentos, escuela, maestro.

\section{ABSTRACT}

Allergic diseases in children are an emergent issue of public health difficult to deal with. nevertheless, there barely are available protocols to identify allergic pupils neither some chapters relative to allergy in childhood and adolescence are included in academic programs of future teachers. Since children spend much time at the school, this project aims to give basic education to future children teachers, directed to know which both asthma and food allergy are, how identify early allergic symptoms, and what to do in front of an allergic episode.

A group of students of university school for children teachers were asked to anonymously fill in a survey about the allergic children at the school, including 44 items. After that, an allergist carried out an educational program of basic allergy directed to the participants, and one month later, the same survey was offered to them in order to size the evolution of the knowledge.

Future children teachers show a low profile of knowledge about allergy. It would be adequate to give them sanitary education about highly prevalent diseases, particularly in the case of pediatric diseases. This project has demonstrated the high value of educational seminars to improve the aimed results.

Key words: Child, allergy, food, school, teacher.

\section{INTRODUCCIÓN}

La alergia en edad pediátrica es un problema de salud pública emergente y a su vez complejo de abordar. Esto es debido a la amplitud, variedad y a los diferentes grados de severidad de las manifestaciones clínicas con que se puede presentar, al elevado número de alérgenos que la originan (alimentos y neumoalérgenos como son los hongos, pólenes, ácaros y epitelios), a las limitaciones diagnósticas existentes y a las complejas medidas terapéuticas que se disponen para su manejo.

Los niños pasan una importante parte de su vida en la escuela, y es sabido por la realización de estudios recientes que un $8 \%$ de los escolares españoles tiene alergia a alimentos y hasta un $20 \%$ de las reacciones alérgicas a alimentos se producen en el entorno escolar. Por ello, es de gran importancia que el profesorado y el personal no docente del centro escolar conozca qué es la alergia, cómo se identifica, cómo actuar en caso necesario y cómo prevenir la recaída de los síntomas alérgicos [1], [2]. 
La atención del niño con alergia en la escuela debe atender a dos premisas, igualmente importantes, su seguridad física y su bienestar emocional. Esto conlleva que la escuela deberá poner en marcha una serie de actuaciones dirigidas a la creación de entornos seguros que garanticen la inclusión del niño en todas las actividades contribuyendo así, a su adecuado desarrollo global atendiendo, especialmente, a su desarrollo emocional.

Las manifestaciones más frecuentes que pueden presentar los niños alérgicos en la escuela son:

- La anafilaxia tras la ingesta de algún alimento durante el recreo ó el comedor (considerada actuación urgente por el riesgo vital y su rápida instauración), ó por la presencia de diferentes alérgenos en muchas actividades escolares. Esta presencia no siempre es evidente ya que se pueden encontrar de forma oculta en la composición de productos de higiene, material escolar ó en alimentos contaminados por haber tenido contacto con el alérgeno [3], [4], [5], [6].

- El asma sobre todo el que tiene lugar durante la primavera en nuestro medio ó el que surge en la clase de Educación Física ó el esfuerzo durante los juegos en el recreo. El broncoespasmo inducido por el ejercicio se define como un estrechamiento transitorio de las vías respiratorias inferiores después del ejercicio en presencia ó ausencia de asma clínicamente reconocida. Tiene una prevalencia que oscila entre un 4-20\% de la población general, siendo del $12 \%$ en niños la de haber presentado en alguna ocasión sensación de sibilancias ("pitos") [7], [8], [9].

En el 2014 se realizó un estudio en el que se pasó un cuestionario (resultados presentados en el Congreso de la Academia Europea de Alergología e Inmunología Clínica-EAACI en Copenhague) [10] sobre conocimientos de alergia alimentaria en el Centro de Magisterio "Sagrado Corazón" de la Universidad de Córdoba. Rellenaron el cuestionario 207 estudiantes universitarios. La puntuación media final sobre conocimiento de la alergia alimentaria fue de 4 puntos, sobre 16. El 31\% creía que la celiaquía es una enfermedad alérgica. El 52\% no sabía que hay alérgicos al huevo que pueden comer yema. Tan solo el $12 \%$ sabía que hay algunos alérgicos al látex que deben evitar las frutas tropicales. El 62\% sabía que hay alérgicos a la piel de la fruta, la cual toleran siempre que la coman sin piel. El 60\% desconocía si los alérgicos a los cereales silvestres pueden comer pan y el $48 \%$ no sabía que la adrenalina puede salvar la vida en una reacción severa. Con los datos anteriores se concluyó que la población encuestada tenía escasos conocimientos sobre la alergia alimentaria, siendo éste un tema cada vez más prevalente en la edad pediátrica y por lo tanto de posible manifestación en la escuela.

Respecto al asma infantil y del adolescente, es bien sabido, la importancia de la educación sanitaria de padres, cuidadores (incluidos los maestros) y del propio niño, según su edad, para su control. La Guía Española del Manejo del Asma (GEMA) menciona que la colaboración mutua entre todos ellos en la educación del paciente asmático, reduce el riesgo de padecer exacerbaciones y aumenta su calidad de vida.

Por ello, dado que la mayoría de los centros educativos en la actualidad, no disponen de personal sanitario, el profesorado debe llevar a cabo una actuación preventiva en la escuela y ser conocedor de las pautas a seguir ante unos síntomas de causa alérgica. Para ello, se precisa: formación en éste tema, identificación del alumnado de riesgo, confección de la ficha técnica de salud actualizada y personalizada de cada niño alérgico, custodia por la escuela de la medicación necesaria actualizada y puesta en marcha de los circuitos de atención urgente y aviso a los padres ó tutor. Concluyendo, éste estudio se plantea tras observar el incremento en los últimos años de sensibilización alérgica en la infancia y a la alta probabilidad que existe de que el niño presente los síntomas alérgicos mientras se encuentra en la escuela y sea, por tanto, el profesor el primero en poder atenderle, lo que hace que tenga una especial relevancia la educación sanitaria en el medio escolar y especialmente a los que en un futuro cercano serán los docentes de los menores.

\section{OBJETIVOS}

* PRINCIPAL:

-Enseñar a estudiantes de Grado en Educación Infantil y Grado en Educación Primaria el protocolo de actuación en una reacción alérgica por alimentos y en la aparición de síntomas de asma en la escuela.

* SECUNDARIOS:

-Mostrar las dificultades en la escolarización del alumnado con alergia a alimentos: situaciones de riesgo. 
-Identificar las diferentes entidades clínicas de las reacciones debidas a alimentos. Saber diferenciar alergia de intolerancia.

-Insistir en el concepto anafilaxia, en la rapidez de instauración y en el protocolo a seguir cuando aparezca. alimentaria.

-Mostrar las diferentes guías que hay para centros educativos y comedores escolares en relación con la alergia

-Adiestrar en el uso de la adrenalina en los casos de anafilaxia. anafilaxia.

-Identificar los primeros síntomas que orientan a una crisis de asma, a una alergia por alimentos ó a una

-Conocer los posibles desencadenantes de una crisis de asma.

-Adiestrar en el manejo de los inhaladores de rescate en los casos de asma. en el asma).

-Enseñar la diferencia entre medidas preventivas y medidas terapéuticas (tanto en la alergia alimentaria como

\section{MATERIAL Y MÉTODOS}

El proyecto se llevó a cabo (Octubre 2017- Mayo 2018) dentro de la asignatura "Bases Biológicas de la Salud" que se imparte en $2^{\circ}$ curso de Grado de la Escuela de Magisterio Sagrado Corazón. Universidad de Córdoba. Los objetivos de dicha asignatura se encuentran especificados en la guía docente, siendo algunos de ellos los siguientes:

- Conocer las técnicas de primeros auxilios y reanimación cardio-pulmonar básica (CE8).

- Adquirir conocimientos básicos para fomentar una alimentación racional en las distintas etapas de la vida (CE8 y CM4.1).

- Saber reconocer las principales enfermedades infantiles con mayor repercusión en el medio escolar para el fomento de la salud y la higiene (CE8, CM4.2 y CM4.4).

- Tomar conciencia de la responsabilidad del maestro en el fomento de la salud y la higiene de la escuela y de los alumnos (CM4.3).

Todos los objetivos expuestos se trabajaron con el proyecto de innovación propuesto, el cual se dividió en tres etapas:

- Una primera etapa (Octubre - Noviembre): Se pasaron diferentes cuestionarios (en formato papel y en línea, como ej: Kahoot) para valorar el conocimiento que tienen los estudiantes de Grado en Educación Infantil y Grado en Educación Primaria, futuros profesores, sobre el asma y la alergia alimentaria, su manifestación más severa que es la anafilaxia y los protocolos que se deben seguir ante una manifestación alérgica en la escuela.

- Una segunda etapa (Diciembre - Marzo): En la que se llevaron a cabo en el aula seminarios y talleres prácticos sobre el asma, la alergia alimentaria y la anafilaxia; manejo del niño alérgico en la escuela en fase aguda, protocolos a seguir ante una reacción alérgica y manejo de inhaladores y de adrenalina.

- El proyecto finalizó con una tercera etapa (Abril - Mayo) en la que se pasó el mismo cuestionario inicial, que nos serviría de evaluación del proyecto de innovación y en el que se comprobaría el aprendizaje adquirido por el alumnado tras los talleres y las sesiones prácticas llevados a cabo en el aula.

También se realizó una evaluación para analizar las dificultades que se pueden encontrar los profesores ante diferentes síntomas de alergia (una vez recibida la formación) y considerar las posibles mejoras.

Para el análisis descriptivo, las variables cuantitativas se presentan con su media y desviación estándar y las variables cualitativas mediante su frecuencia y porcentaje. Se utiliza la prueba de contraste t de Student para la comparación de dos medias, en el caso de puntuación del examen antes y después; y en el caso de las variables cualitativas se ha aplicado la prueba de ji-cuadrado. Hemos considerado un nivel de significación p<0,05.

\section{RESULTADOS OBTENIDOS}


En una primera sesión llevada a cabo en Noviembre, tras exponer brevemente el tema del niño alérgico en la escuela, se le pidió a los participantes que expusieran de forma informal, libre y por escrito (Tormenta-Llwiva de ideas) su opinión sobre el tema.

Contestaron 40 alumnos de Grado de Infantil. 100\% mujeres.

- El 90\% definió el tema como muy interesante.

- El 100\% como un tema que era importante y muy práctico para su futuro como maestros y en el que debían estar bien formados.

- El 30\% manifestó que el maestro podía ser una gran ayuda para el niño si tenía lugar una reacción alérgica en la escuela. Y, además, destacaban la responsabilidad del maestro en la escuela.

En una segunda sesión se propuso a los participantes (diferentes a los que participaron en la Tormenta de Ideas) que rellenaran de forma anónima un cuestionario donde se preguntaba acerca de aspectos sociodemográficos básicos (edad, sexo, presentar alergia, hábitat), y diferentes preguntas acerca de la alergia (bloque general- 5 preguntas, bloque de alergia respiratoria- 6 , alimentaria-10, a medicamentos- 2 , a himenópteros-4, de tratamiento-adrenalina e inhaladores4 y de neumoalergenos-13), mediante 44 preguntas con 3 opciones (verdadero, falso, no contesta) y una sola respuesta correcta, siendo valorada con +1 la respuesta correcta. Después se extrapolaría sobre 10.

Rellenaron el cuestionario 75 alumnos (42 varones, 56\%), con una edad media de 21 años. El 65\% vive de forma habitual en medio urbano. $24(32 \%)$ se consideraban alérgicos y el $47 \%$ de estos habían sido vistos por este motivo por un médico. Tan solo 11 estaban con inmunoterapia. $53(71 \%)$ consideraban bastante interesante el tema de la alergia. $48 \%$ buscan en Internet las dudas sobre la salud y $48 \%$ preguntan a un médico. La puntuación media final fue de 4.2 puntos sobre 10. Tan sólo obtuvieron un cinco ó más de cinco 21 personas (28\%). Es decir, el 72\% de los encuestados suspenden.

También se llevó a cabo un Kahoot con 15 preguntas alergológicas a 75 alumnos. El 68\% de los alumnos contestaron erróneamente ó fuera de tiempo.

En una tercera sesión, se llevaron a cabo diferentes talleres formativos en grupos de no más de 30 personas. Se les recomendó a los alumnos, que no tomaran nota. Tan sólo que participaran y que prestaran atención. Se explicó y se interpretó como puede presentar un niño una reacción alérgica en la escuela, se mostró y se enseñó el manejo de la adrenalina autoinyectable, se insistió que precauciones se deben tomar en el recreo y durante la clase de Educación Física, y durante el comedor y las celebraciones en el horario escolar. Se explicó lo que es el asma y los diferentes inhaladores que puede precisar un niño.

En una cuarta sesión, a los 40 días de haber recibido la formación en los talleres, se les volvió a pasar a los alumnos el cuestionario de las 44 preguntas y lo volvieron a contestar. De nuevo lo contestaron los 75 alumnos. La nota definitiva fue de 6.5 sobre 10 ( $\mathrm{p}<0,001)$ y aprobaron el $90.4 \%$, suspendiendo tan sólo el $9.6 \%(\mathrm{p}<0,001)$.

En una quinta sesión se les preguntó a los alumnos (30) que opinaban de la formación del manejo en la escuela del niño alérgico que habían recibido durante el año. El 100\% se sentía muy satisfecho con lo aprendido, insistían en la utilidad de lo aprendido y pedían más formación en el futuro en temas de salud aplicables en la escuela.

\section{DISCUSIÓN}

La atención del niño con alergia en la escuela debe atender a su seguridad física dentro de su entorno cotidiano. Esto conlleva que la escuela deberá poner en marcha una serie de actuaciones dirigidas a la creación de entornos seguros que garanticen la inclusión del niño en todas las actividades contribuyendo así, a su adecuado desarrollo global atendiendo, especialmente, a su desarrollo emocional.

Los niños pasan una importante parte de su vida en la escuela, aproximadamente un $30 \%$ de su tiempo durante el curso escolar, por lo que es altamente probable que el niño alérgico presente asma ó una reacción alérgica por alimentos en el entorno escolar.

Se ha elaborado un documento consensuado entre el Ministerio de Educación, Cultura y Deporte, el Ministerio de Sanidad, Servicios Sociales e Igualdad, y las Consejerías y/ó Departamentos de Educación y de Sanidad 
de las Comunidades Autónomas, en el que se recogen una serie de recomendaciones para una escolarización segura e igualitaria del alumnado susceptible de sufrir alergia a los alimentos. Aunque la prevención es una medida mucho más útil que la actuación ante una emergencia, este documento da especial importancia a:

- La información/formación del personal del centro escolar en el tema.

- La elaboración de un Plan de Actuación para el alumnado alérgico.

- La propuesta de un protocolo de intervención ante una reacción alérgica.

Con este proyecto se muestra que los futuros maestros tienen escasos conocimientos del manejo en la escuela de los niños alérgicos. Pero también se demuestra que la realización de seminarios informativos mejora de forma muy significativa el conocimiento sobre dicho tema. También los alumnos son conscientes de la importancia del tema y de la necesidad de información y formación al respecto. Por ello, debería valorarse en un futuro, incluir en el Plan Docente formación en estos temas de salud.

En años sucesivos se impartirán los seminarios a los nuevos alumnos y se debería hacer un recordatorio a los que ya hayan recibido la formación, especialmente a los cursos que terminen su formación académica.

\section{CONCLUSIONES}

A pesar que la formación de los estudiantes de Grado en Educación Infantil y Grado en Educación Primaria, futuros maestros, en nociones básicas sobre qué es y qué supone una alergia alimentaria y/ó un asma, en las medidas preventivas con el fin de evitar reacciones, en el reconocimiento de los primeros síntomas de una reacción alérgica y en cómo actuar cuando ésta se ha producido (hincapié en el manejo del autoinyector de adrenalina y en el uso de los inhaladores de rescate) mientras el niño se encuentra en el colegio, es muy escasa, se muestra con el trabajo presentado la utilidad e importancia en la formación en estos temas.

AGRADECIMIENTOS

Al Centro Universitario de Magisterio Sagrado Corazón. Universidad de Córdoba. Por facilitar y ayudar en el desarrollo de este proyecto.

\section{BIBLIOGRAFÍA}

1. Fong AT, Katelaris CH. Bullying and quality of life in children and adolescents with food allergy. Journal of Paediatrics and Child Health. Vol 53, Issue 7, July 2017. P 630-5.

2. Estudio Alergológica 2015. Pág: 318. ISBN: 978-84-88014-41-2.

3. Sampson HA, Muñoz A, Campbell RL, Adkinson NF, Allan S, et al. Second Symposium on the definition and management of anaphylaxis: Sumary report-Second National Institute of Allergy and Infectious Disease/Food Allergy and Anaphylaxis Network Symposium. Ann Emerg Med. 2006; 47: 373-80.

4. Grabenhenrich LB, Dolle S, Moneret A, et al. Anaphylaxis in children and adolescents: The European Anaphylaxis Registry. J Allergy Clin Immunol. 2016 Apr; 137 (4): 1128-37.

5. Murano A, Roberts G, Worm M, et al. Anaphylaxis: guidelines from the European Academy of Allergy and Clinical Immunology. Allergy 2014: 69: 1026-45.

6. Juliá JC, Sánchez CA, Alvarado MI, et al. Manual de Anafilaxia pediátrica. SEICAP. 2017. ISBN: 978-84-617-96045

7. Dávila I, Jáuregui I, Olaguibel JM, Zubeldia JM. Tratado de Alergología. Tomo II. $2^{a}$ Edición. 2015. ISBN: 978-8416270-38-5. 
8. Executive Committee GEMA 2009. Gema 2009 (Spanish guideline on the management of asthma). J Investig Allergol Clin Immunol. 2010; 20 (Suppl 1): 1-59.

9. Guía GEMA 4.1. Disponible en http://www.gemasma.com

10. Barasona MJ, Barasona ML, García I, Algaba MM, Moreno C. Food Allergy: a world unknown to the general population. Allergy. 2014; 69. (Supl 99): 282-3. 